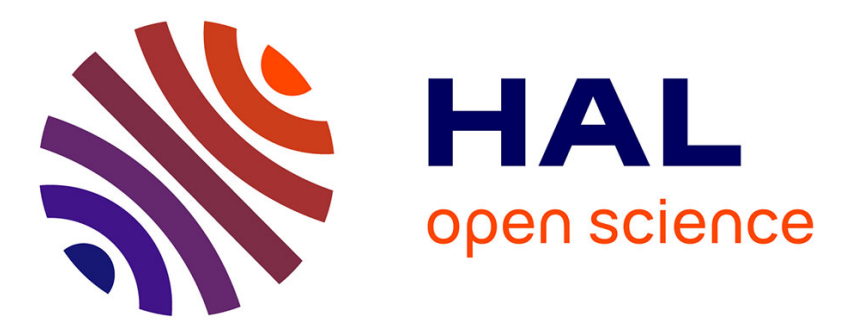

\title{
Prédiction par simulation des défauts de plissement lors de la mise en forme des matériaux composites mono et multiplis
}

\author{
Peng Wang, Nahiene Hamila, Philippe Boisse
}

\section{- To cite this version:}

Peng Wang, Nahiene Hamila, Philippe Boisse. Prédiction par simulation des défauts de plissement lors de la mise en forme des matériaux composites mono et multiplis. 17èmes Journées Nationales sur les Composites (JNC17), Jun 2011, Poitiers-Futuroscope, France. pp.149. hal-00598122

\section{HAL Id: hal-00598122 \\ https://hal.science/hal-00598122}

Submitted on 4 Jun 2011

HAL is a multi-disciplinary open access archive for the deposit and dissemination of scientific research documents, whether they are published or not. The documents may come from teaching and research institutions in France or abroad, or from public or private research centers.
L'archive ouverte pluridisciplinaire HAL, est destinée au dépôt et à la diffusion de documents scientifiques de niveau recherche, publiés ou non, émanant des établissements d'enseignement et de recherche français ou étrangers, des laboratoires publics ou privés. 


\title{
Prédiction par simulation des défauts de plissement lors de la mise en forme des matériaux composites mono et multiplis
}

\author{
Numerical prediction of wrinkling defects during single and multi layered \\ composite forming process
}

\author{
Peng Wang ${ }^{1}$, Nahiene Hamila ${ }^{1}$ et Philippe Boisse ${ }^{1}$ \\ 1 : Laboratoire de Mécanique des Contacts et des Structures \\ Institut National des Sciences Appliquées de Lyon \\ Bâtiment Jacquard \\ 27 avenur Jean Capelle \\ F69621 VILLEURBANNE CEDEX \\ e-mail : peng.wang@insa-lyon.fr,nahiene.hamila@insa-lyon.fr, philippe.boisse@insa-lyon.fr
}

\begin{abstract}
Résumé
Lors de la mise en forme des renforts de composites l'apparition des plissements fait parti des défauts les plus courants. Ces plissements sont fréquents en raison des très faibles raideurs en flexion dues aux mouvements relatifs entre les fibres constituant chaque mèche. Il est nécessaire de détecter leur apparition, ainsi que leur évolution au cours de la phase de préformage, et ce afin de s'assurer qu'ils ne s'étendent pas à la partie utile de la pièce. La simulation des renforts de composites textiles en cours de mise en forme se fonde sur une forme simplifiée du travail virtuel des efforts internes de chaque cellule élémentaire, découplée en une énergie de tension, de cisaillement dans le plan et du moment fléchissant. L'influence des trois rigidités (traction, cisaillement dans le plan et flexion) sur l'apparition des plissements lors de la simulation est analysée. Même si la raideur en cisaillement dans le plan joue un rôle principal sur leur apparition, il n'y a pas de corrélation directe entre l'angle de cisaillement et les plis. Le plissement est un phénomène global dépendant autant des déformations, des raideurs et des conditions aux limites. La raideur en flexion joue un rôle principal sur la forme que prendront ces plis, et il n'est pas possible de simuler proprement un pli avec une approche membranaire seule.
\end{abstract}

\begin{abstract}
Wrinkling is one of the most common flaws that occur during textile composite reinforcement forming processes. These wrinkles are frequent because of the possible relative motion of fibres making up the reinforcement, leading to a very weak textile bending stiffness. It is necessary to simulate their onset but also their growth and their shape in order to verify that they don't extend to the useful part of the preform. In this paper the simulation of textile composite reinforcement forming and wrinkling is based on a simplified form of virtual internal work defined according to tensions, in-plane shear and bending moments on a unit woven cell. The role of the three rigidities (tensile, in-plane shear and bending) in wrinkling simulations is analysed. If in-plane shear stiffness plays a main role for onset of wrinkles in double-curved shape forming, there is no direct relation between shear angle and wrinkling. Wrinkling is a global phenomenon depending on all strains and stiffnesses and on boundary conditions. The bending stiffness mainly determines the shape of the wrinkles and it is not possible to perform a wrinkle simulation using a membrane approach.
\end{abstract}

Mots Clés : Renfort textile, analyse par éléments finis, flambage, plissements

Keywords : Fabrics/textiles, Finite element analysis, Buckling, Wrinkling

\section{Introduction}

Pour la fabrication de textile composite sur des formes non-développables, le drapage de préimprégnés ou de tissus secs est nécessaire. Dans le cas des renforts secs, le procédé de moulage par injection (LCM) consiste à donner la forme de la pièce finale au renfort puis à y injecter la résine [1, 2]. Le renfort textile peu subir de fortes déformations, et de grands angles de cisaillement dans le plan peuvent s'avérer être nécessaires pour obtenir la forme désirée. Selon la géométrie des pièces, le type de renfort (tissage, matière ...) et les paramètres de fabrication (chargements des outils, serre-flans...), les formes à double courbure peuvent comporter des défauts. Des logiciels de 
simulation pour le formage des composites ont été développés pour déterminer les paramètres de faisabilité du procédé [3-6]. Les plissements sont parmi les défauts les plus courants qui se produisent sur les renforts textiles. Ces plis se forment facilement en raison de la composition des mèches faites de fibres. Le mouvement relatif des fibres entre elle conduit à un comportement mécanique très spécifique aux renforts tissés. Cela lui fournit une très faible résistance à la flexion qui le rend propice à l'apparition des plissements.

Les plis sont un problème majeur à traiter lors de la conception des pièces minces et de la définition des paramètres de formage du renfort. Une grande partie des efforts de recherche a été déployé afin de les prédire dans un cadre isotrope [7-10] et anisotrope [11, 12]. Dans le cas particulier des renforts textiles, la structure interne du tissu faite de mèches et de fibres rend le phénomène de plissement fréquent. Certaines études expérimentales de cisaillement dans le plan ont analysé les plissements, se concentrant sur l'angle de blocage en cisaillement [13-18]. Une analyse numérique de l'apparition des plis a été proposée en se basant principalement sur des modèles cinématiques (aussi appelé algorithmes du filet) [19-21], des représentations simplifiées de treillis [22], ou encore sur des approches membranaires [23-25].

Cet article examine une forme simplifiée du travail virtuel interne dans le cas des composites à renforts textiles. Le travail virtuel en tension, cisaillement dans le plan et en flexion de chaque cellule élémentaire sont découplés. La forme de ces travaux virtuels est simple et proche de la physique des milieux textiles. Les données matérielles impliquées sont directement identifiés par des tests mécaniques établis pour le cas de renforts textiles. Les essais mécaniques comprennent des tests de tractions biaxiales pour la rigidité en tension [26-29], de picture frame et de bias-test pour la rigidité de cisaillement dans le plan [13-18, 30-36] et des KES pour la flexion [37-39].

Un élément fini de coque à trois nœuds, spécifique aux milieux tissés, basé sur cette forme de travail virtuel a été développé $[40,41]$. Il est utilisé pour simuler le drapage des renforts textiles et pour leur formage afin d'analyser l'apparition et l'évolution des plis en fonction des trois raideurs (tension, en cisaillement et de flexion).

Les plis peuvent être dus à la compression dans la direction des fils (ce qui est généralement évitée dans le processus de formage à l'aide des serre-flans) ou aux grandes déformations en cisaillement plan. La rigidité de cisaillement dans le plan d'un tissu augmente fortement avec l'angle de cisaillement qui atteint un 'angle de blocage'. Il est cependant difficile d'établir une relation simple entre le cisaillement et l'apparition des défauts.

La raideur en flexion joue un rôle important sur la forme des plis. Une augmentation de cette rigidité conduit à une augmentation de leur taille. Pour certains procédés de formage, il y a des zones où les plis ne peuvent être évités. Dans ces cas, il est important de simuler les formes de ces plis afin de vérifier qu'ils ne s'étendent pas à la partie utile de la préforme. Il n'est pas convenable d'adopter des approches membranaires communément utilisées pour les simulations de drapage des renforts textiles. Pour effectuer une simulation efficace il est nécessaire de prendre en compte tant les tensions des mèches, que le cisaillement dans le plan et les énergies de déformation en flexion.

\section{Comportement mécanique des renforts de composites textiles}

\subsection{Décomposition des raideurs d'une cellule élémentaire représentative}

Le renfort textile composite est constitué de cellules élémentaires représentatives (CER). Ce sont des motifs géométriques, qui par simple translation, permettent de représenter l'ensemble du tissage. Le comportement mécanique du tissu est spécifique en raison des déplacements relatifs possibles entre les mèches, et des déplacements relatifs entre les fibres à l'intérieur de ces mèches. Examinons les chargements sur une cellule unitaire de tissé comme Fig. 1a. Les résultantes suivantes sont envisagées:

- Les tensions $T_{1}$ et $T_{2}$ sont les résultantes des tensions respectivement sur les mèches chaînes et de trame, duales des déformations dans la direction $\underline{\mathrm{f}}_{1}$ et $\underline{\mathrm{f}}_{2}$ des mèches (Fig. 1b) 
- Le moment dans le plan de cisaillement $\mathrm{M}_{\mathrm{s}}$, le moment au centre de la CER selon la normale à la surface du tissu, résultant de l'interaction entre les deux réseaux de mèches. Il s'agit ici du dual de l'angle de cisaillement entre chaîne et trame (Fig. 1c).

- $\quad$ Les moments de flexion $\mathrm{M}_{1}$ et $\mathrm{M}_{2}$ sur la chaîne et la trame, grandeurs duales des courbures dans les deux directions (Fig. 1d).
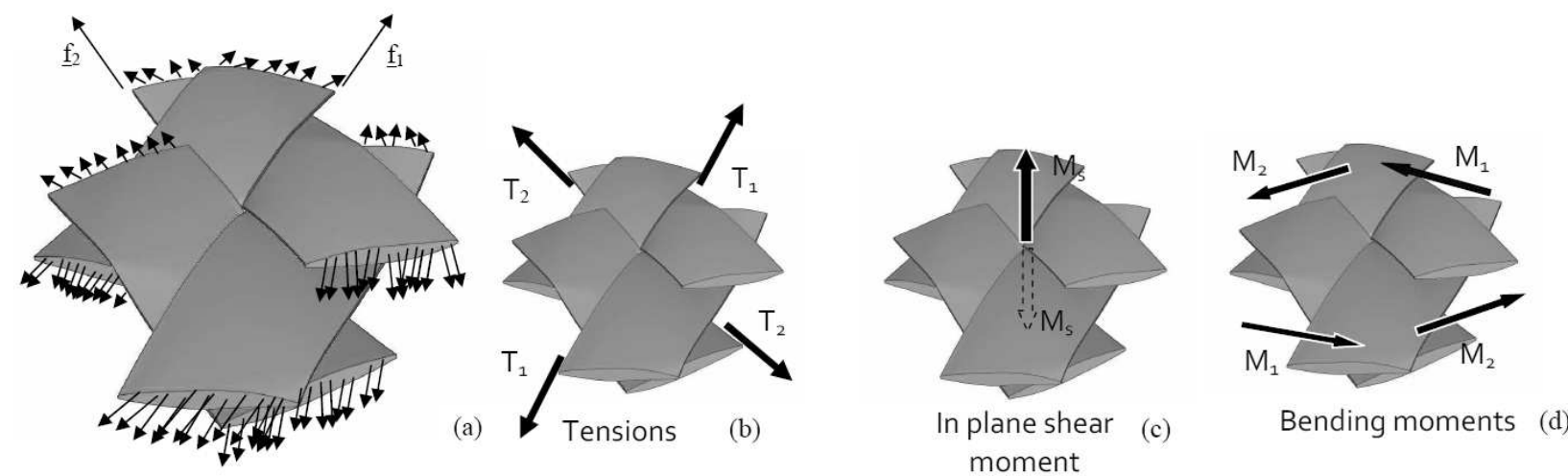

Fig. 1. (a) Chargement sur une CER et résultantes en effort: (b) tensions, (c) cisaillement dans le plan, (d) moments de flexion.

Il s'agit d'une modélisation simplifiée, mais le sens physique de ces grandeurs est quantifiable. En effet, elles sont directement mesurées en fonction de la déformation du tissu lors de tests standard de renforts composites. Enfin, ces charges $\mathrm{T}_{1}, \mathrm{~T}_{2}, \mathrm{M}_{\mathrm{s}}, \mathrm{M}_{1}$ et $\mathrm{M}_{2}$ sont respectivement conjuguées aux déformations axiales $\varepsilon_{11}$ et $\varepsilon_{22}$ en sens chaîne et trame, à $\gamma$ l'angle de cisaillement dans le plan, et des courbures $\chi_{11}$ et $\chi_{22}$ dans le sens chaîne et trame. Cela conduit à la forme simple du travail virtuel interne présentée ci-dessous.

\subsection{Principe des puissances virtuelles}

Un renfort textile composite est constitué de $\mathrm{N}_{\mathrm{c}}$ cellules élémentaires similaires à celles représentées figure. 1. Ces schémas ont été dessinés dans le cas particulier d'un satin pour plus de simplicité, mais le type de tissage peut être différent. Le principe de travail virtuel peut être écrit:

$$
\begin{aligned}
& \forall \underline{\eta} / \underline{\eta}=0 \text { on } \Gamma_{\mathrm{u}}, \quad \mathrm{W}_{\mathrm{ext}}(\underline{\eta})-\mathrm{W}_{\mathrm{acc}}(\underline{\eta})= \\
& \sum_{\mathrm{p}=1}^{\mathrm{N}_{\mathrm{c}}} \underbrace{{ }^{\mathrm{p}} \varepsilon_{11}(\underline{\eta}){ }^{\mathrm{p}} \mathrm{T}_{1}{ }^{\mathrm{p}} \mathrm{L}_{1}+{ }^{\mathrm{p}} \varepsilon_{22}(\underline{\eta}){ }^{\mathrm{p}} \mathrm{T}_{2}{ }^{\mathrm{p}} \mathrm{L}_{2}}_{\text {(b) }}+\underbrace{\gamma(\underline{\eta}){ }^{\mathrm{p}} \mathrm{M}_{\mathrm{s}}}_{\text {(c) }}+\underbrace{{ }^{\mathrm{p}} \chi_{11}(\underline{\eta}){ }^{\mathrm{p}} \mathrm{M}_{1}{ }^{\mathrm{p}} \mathrm{L}_{1}+{ }^{\mathrm{p}} \chi_{22}(\underline{\eta}){ }^{\mathrm{p}} \mathrm{M}_{2}{ }^{\mathrm{p}} \mathrm{L}_{2}}_{\text {(d) }}
\end{aligned}
$$

$\mathrm{W}_{\text {ext }}(\underline{\eta})$ et $\mathrm{W}_{\mathrm{acc}}(\underline{\eta})$ sont respectivement le travail virtuel des efforts externe et le travail virtuel des forces d'accélération. $\underline{\eta}$ est le champ de déplacement virtuel égal à zéro là où les déplacements sont imposés. La quantité A est notée ${ }^{\mathrm{p}} \mathrm{A}$ quand elle est associée à la CER p. La partie (b) des travaux virtuel interne est due à des tensions. $\varepsilon_{11}(\underline{\eta})$ et $\varepsilon_{22}(\underline{\eta})$ sont les déformations virtuelles dues à l'allongement des mèches dans le sens chaîne et trame. $\mathrm{L}_{1}$ et $\mathrm{L}_{2}$ sont les longueurs de la mèche dans le sens chaîne et dans le sens trame. La partie (c) représente le travail virtuel interne dû au cisaillement dans le plan. $\gamma(\underline{\eta})$ est la variation d'angle virtuel entre les directions chaîne et trame sous le champ de déplacement $\underline{\underline{\eta}}$. La partie (d) est le travail virtuel interne du à la flexion. $\chi_{11}(\underline{\eta})$ et $\chi_{22}(\underline{\eta})$ sont les courbures virtuelles dans des directions chaîne et trame. Les grandeurs cinématiques virtuelles $\varepsilon_{11}(\underline{\eta}), \varepsilon_{22}(\underline{\eta}), \gamma(\underline{\eta}), \chi_{11}(\underline{\eta})$ et $\chi_{22}(\underline{\eta})$ sont uniquement fonctions du champ de déplacement virtuel $\underline{\eta}$.

\section{Elément fini semi discret de coque pour les renforts de composite}


L'élément fini de coque à trois nœuds utilisé dans ce document est basé sur la forme simplifiée du principe des travaux virtuels donnée dans l'équation 1. Les détails de sa formulation sont donnés dans $[40,41]$. Elle est résumée ci-dessous. Les raideurs en tension, en cisaillement dans le plan et en flexion sont séparées afin d'analyser leurs influences sur les simulations des plissements.

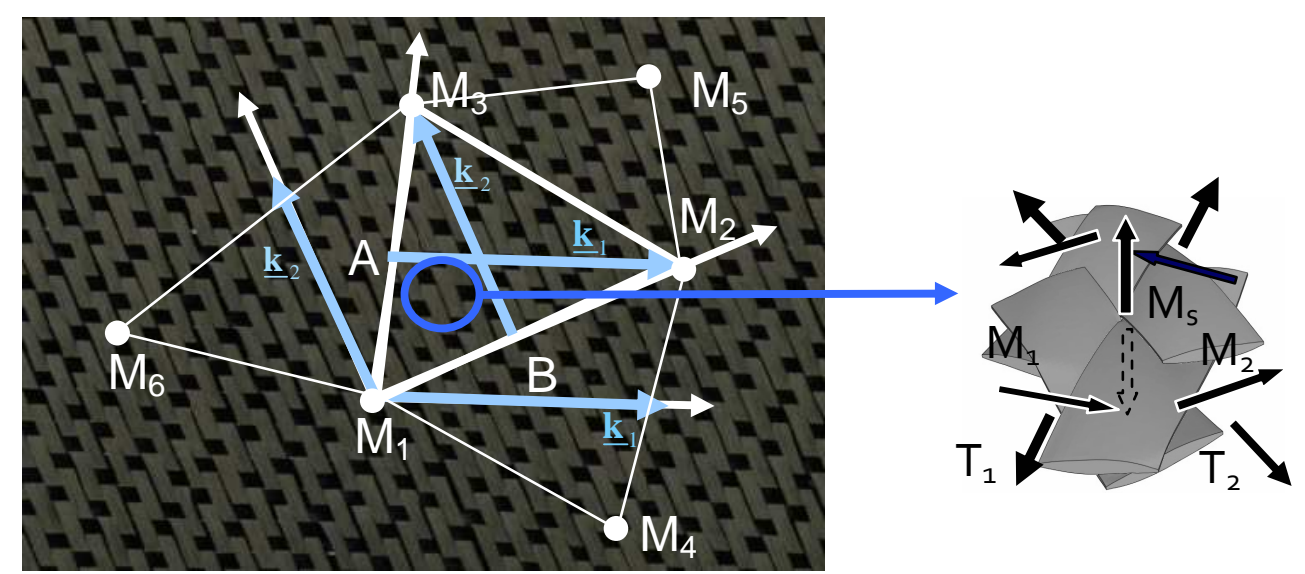

Fig. 2. Elément fini triangulaire composé de cellules élémentaires.

Un élément fini de coque à trois noeuds $\mathrm{M}_{1} \mathrm{M}_{2} \mathrm{M}_{3}$ composé de ncelle CER de tissé est considéré (Fig. 2). Les vecteurs $\mathrm{k}_{1}=\mathrm{AM}_{2}$ et $\mathrm{k}_{2}=\mathrm{BM}_{3}$ respectivement dans les directions de chaîne et de trame sont définis. Le travail virtuel interne dans l'élément dû aux tensions des mèches (partie (b) dans l'équation 1) définit les forces nodales élémentaires $\mathbf{F}_{\text {int }}^{\text {te }}$ à l'aide de l'expression suivante:

$$
\sum_{\mathrm{p}=1}^{\mathrm{ncelle}}{ }^{\mathrm{p}} \varepsilon_{11} \underline{(\underline{\eta})}{ }^{\mathrm{p}} \mathrm{T}_{1}{ }^{\mathrm{p}} \mathrm{L}_{1}+{ }^{\mathrm{p}} \boldsymbol{\varepsilon}_{22}(\underline{\eta}){ }^{\mathrm{p}} \mathrm{T}_{2}{ }^{\mathrm{p}} \mathrm{L}_{2}=\boldsymbol{\eta}^{\mathrm{eT}} \mathbf{F}_{\text {int }}^{\mathrm{te}}
$$

Les composants internes des résultantes en tension sont calculées à partir des tensions T1 et T2:

$$
\left(\mathrm{F}_{\mathrm{int}}^{\mathrm{te}}\right)_{\mathrm{ij}}=\operatorname{ncelle}\left(\mathrm{B}_{\mathrm{ijj}} \mathrm{T}_{1} \frac{\mathrm{L}_{1}}{\left\|\underline{\mathrm{k}}_{1}\right\|^{2}}+\mathrm{B}_{2 \mathrm{ij}} \mathrm{T}_{2} \frac{\mathrm{L}_{2}}{\left\|\underline{\mathrm{k}}_{2}\right\|^{2}}\right)
$$

i est l'indice de la dimension dans l'espace ( $\mathrm{i}=1$ à 3$)$, $\mathrm{j}$ est l'indice du noeud ( $\mathrm{j}=1$ à 3 ). $\mathrm{B}_{1 \mathrm{ij}}$ et $\mathrm{B}_{2 \mathrm{ij}}$ constituent l'interpolation des déformations en élongations des mèches. Ils sont constants sur l'élément car les fonctions d'interpolation des triangles à trois nœuds, sont linéaires. Le travail virtuel interne en cisaillement dans le plan de l'élément (partie (c) dans l'équation 1) définit les forces internes élémentaires $\mathbf{F}_{\text {int }}^{\text {se }}$ :

$$
\sum_{\mathrm{p}=1}^{\mathrm{ncelle}}{ }^{\mathrm{p}} \gamma(\underline{\eta}){ }^{\mathrm{p}} \mathrm{M}_{\mathrm{s}}=\boldsymbol{\eta}^{\mathrm{eT}} \mathbf{F}_{\mathrm{int}}^{\mathrm{se}}
$$

Les composantes des forces internes dues au cisaillement dans le plan sont calculées à partir du couple de cisaillement:

$$
\left(\mathrm{F}_{\text {int }}^{\mathrm{se}}\right)_{\mathrm{ij}}=\text { ncelle } \mathrm{B}_{\gamma \mathrm{ij}} \mathrm{M}^{\mathrm{s}}(\gamma)
$$

Afin d'éviter d'augmenter le nombre de degrés de liberté et par conséquent l'efficacité numérique, la raideur en flexion est traitée à l'aide d'une approche sans degrés de liberté en rotation [42, 43]. Pour se faire, les courbures de l'élément sont calculées à partir des positions et des déplacements des noeuds des éléments voisins (Fig. 2). Le travail virtuel interne en flexion de l'élément (partie (d) dans l'équation 1) permet d'exprimer les forces internes élémentaires $\mathbf{F}_{\text {int }}^{\text {be }}$ : 


$$
\sum_{\mathrm{p}=1}^{\text {ncelle }}{ }^{\mathrm{p}} \chi_{11}(\underline{\eta}){ }^{\mathrm{p}} \mathrm{M}_{1}{ }^{\mathrm{p}} \mathrm{L}_{1}+{ }^{\mathrm{p}} \chi_{22}(\underline{\eta}){ }^{\mathrm{p}} \mathrm{M}_{2}{ }^{\mathrm{p}} \mathrm{L}_{2}=\boldsymbol{\eta}^{\mathrm{eT}} \mathbf{F}_{\text {int }}^{\mathrm{be}}
$$

Les composantes de ces forces internes en flexion sont calculées à partir des moments de flexion $\mathrm{M}_{1}$ et $\mathrm{M}_{2}$ :

$$
\left(F_{\text {int }}^{\text {be }}\right)_{\mathrm{km}}=\mathrm{n}_{\text {celle }}\left(B b_{1 \mathrm{~km}} \mathrm{M}_{1} \frac{\mathrm{L}_{1}}{\left\|\underline{\mathrm{k}}_{1}\right\|^{2}}+\mathrm{Bb}_{2 \mathrm{~km}} \mathrm{M}_{2} \frac{\mathrm{L}_{2}}{\left\|\underline{\mathrm{k}}_{2}\right\|^{2}}\right)
$$

\section{Analyse par simulation de l'influence sur les plissements des différentes raideurs}

\subsection{Drapage sur une forme hémisphérique}

Le drapage d'un renfort tissé sur un hémisphère est traité (Fig. 3). La géométrie initiale du tissu est carrée de dimension $150 \mathrm{~mm}$ x $150 \mathrm{~mm}$. Le diamètre de l'hémisphère est de $100 \mathrm{~mm}$. La rigidité en tension du tissu est de 1000 N/fils dans les sens chaîne et trame. Le couple de cisaillement dans le plan est exprimé sous la forme polynomiale $\mathrm{M}_{\mathrm{s}}(\gamma)=0.37044 \gamma+0.84105 \gamma^{3}+1.03113 \gamma^{5}$.

La première forme déformée (Fig. 3b) ne prend en compte que (b) en tension de l'équation 1 . Le drapage est obtenu avec une absence totale de plissement, mais les angles de cisaillement proche des coins du tissu avoisinent les $90^{\circ}$. La solution obtenue est similaire à celle donnée par un algorithme du filet. Seules les déformations dans la direction des mèches dues aux tensions dans les fils peuvent entraîner une différence. Mais ces déformations sont très faibles dans le cas présent à cause des faibles tensions au cours du drapage. La deuxième forme (Fig. 3c) est obtenue lorsque les parties en tension (b) et en cisaillement plan (c) du travail virtuel interne de l'équation (1) sont prises en compte. De nombreux plissement de petites tailles sont présents. La troisième forme déformée (Fig. 3d) est obtenue en prenant en compte toutes les composantes de l'énergie (en tension (b), en cisaillement dans le plan (c) et en flexion (d)). Dans ce cas aussi, le drapage conduit à des plissements moins nombreux et ayant des formes plus réalistes.

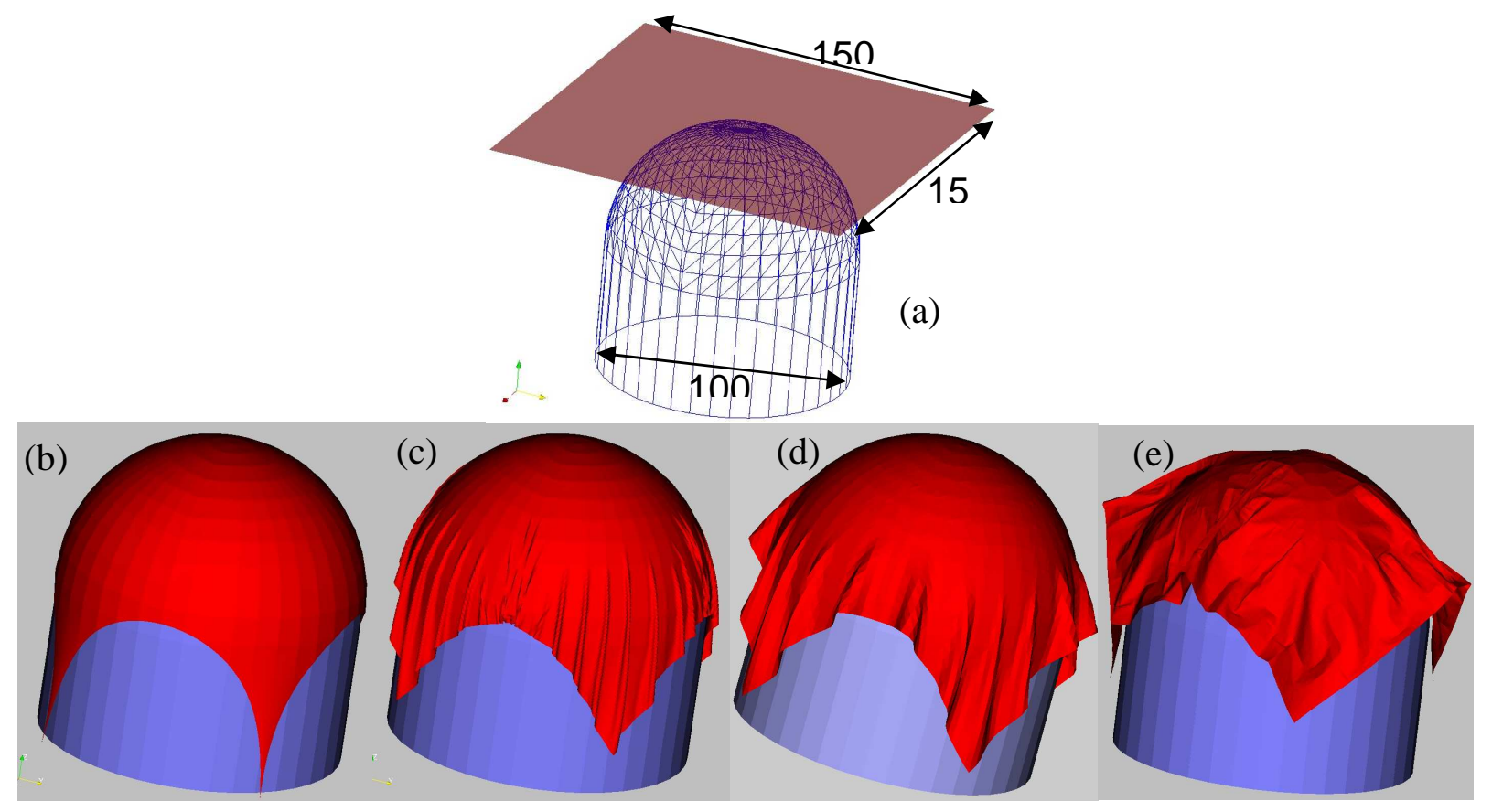

Fig 3. Drapage sur un hémisphère, (a) géométrie initiale, (b) raideur de tension seule, (c) raideur en tension et cisaillement dans le plan, (d) traction - cisaillement plan + raideur en flexion, (e) membrane isotrope.

4.2 Mise en forme d'un renfort déséquilibré. 
Le renfort textile présenté figure 4 est très déséquilibrée. Les propriétés mécaniques mesurées montrent des raideurs de tension égales à $50 \mathrm{~N}$ dans le sens chaîne et $0,2 \mathrm{~N}$ dans le sens de la trame [44]. Le dispositif expérimental de formation par un poinçon hémisphérique a été réalisé à l'Université de Nottingham. [44]. Afin d'éviter l'apparition de plissements dans la zone utile, un anneau de $6 \mathrm{~kg}$ a été utilisée comme serre flanc. La forme expérimentale obtenue après mise en forme est représentée sur la figure 4e. Dans le sens de la chaîne (avec la plus forte rigidité) un fort avalement du renfort est observé. Par contre, dans le sens de la trame (sens faible) aucun mouvement de bord est représenté et les mèches sont soumises à de grandes déformations.

Près du point central de la préforme, un carré dessiné initialement sur le tissu se transforme en un rectangle ayant un rapport longueur / largeur de 1,8 (voir la figure 4e). Les formes simulées après le formage sont montrées Fig. 4b, c, d. Dans la figure. 4b, la raideur en tension est prise en compte (partie (b) dans l'équation 1). La dissymétrie de la forme en sens chaîne et trame est correctement obtenues, mais il n'y apparaît aucun plissement. Fig. 4c montre la forme déformée calculée en prenant en compte les énergies de déformation en cisaillement (mais pas de raideur en flexion). Un grand nombre de petits plissements apparaît. Lorsque l'énergie de déformation en flexion est ajoutée (figure 4d), les plis sont beaucoup plus larges et leurs formes générales sont en assez bon accord avec l'expérience. Il convient de noter que, si la forme et le nombre de plis sont très différents dans les trois simulations, le rapport d'extension au centre de l'hémisphère (trame / chaîne $=1,8$ ) est correctement calculé dans les trois cas. Ce rapport dépend de la raideur en tension prise en compte dans les trois cas. En revanche un algorithme du filet, qui ne prend pas en compte les propriétés mécaniques, conduirait à la même déformation dans les deux sens chaîne et trame, et le rapport dans la partie centrale resterait égal à 1 .
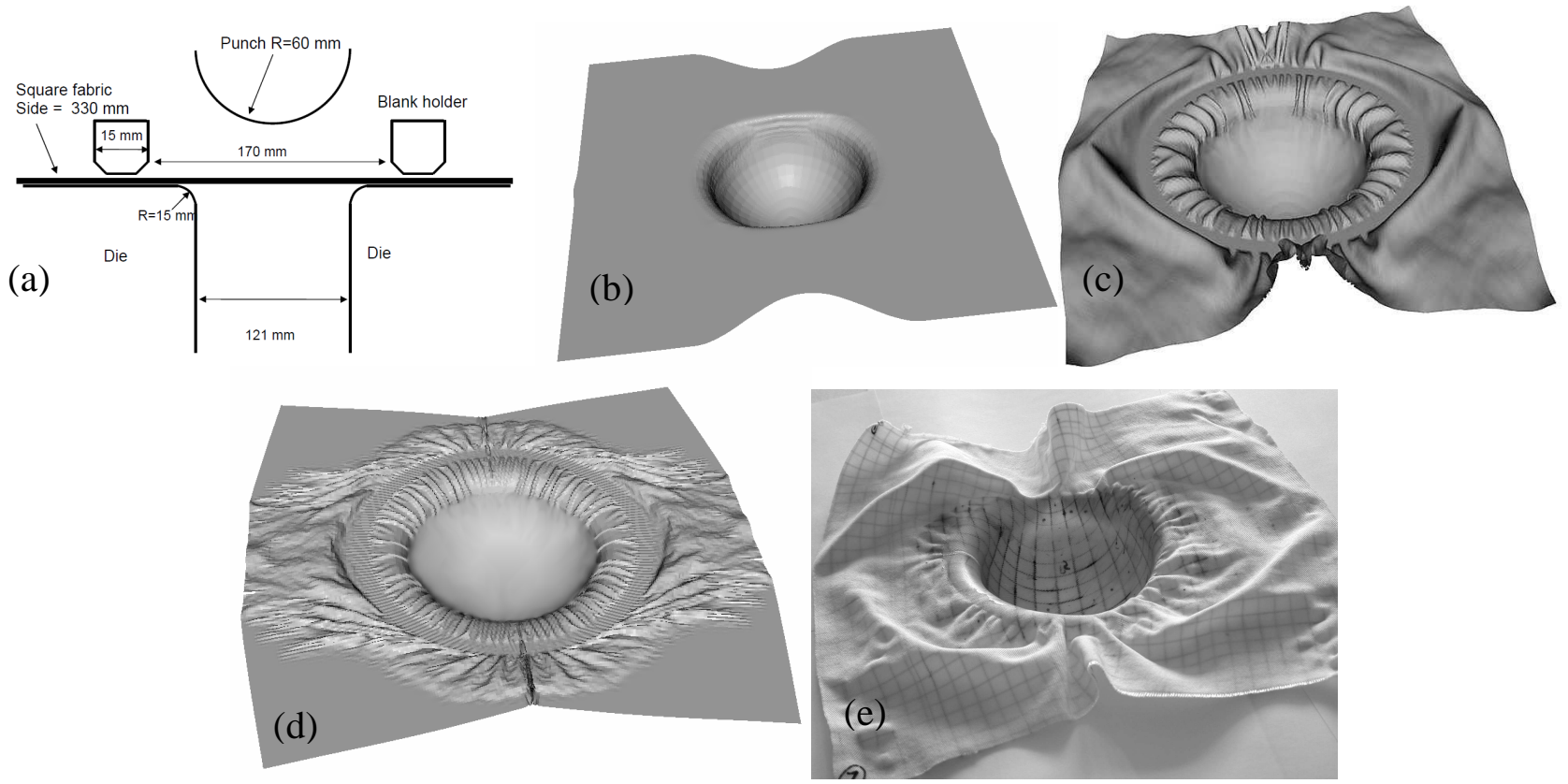

Fig. 4. Mise en forme d'un renfort textile déséquilibré, (a)géométrie des outils,(b)raideur en tension seule,(c)raideur en tension et en cisaillement, (d)tension+cisaillement +flexion, (e)résultat expérimental.

\subsection{Mise en forme à l'aide d'un poinçon cylindrique.}

La simulation de l'emboutissage d'un renfort tissé avec un poinçon cylindrique est présentée Fig. 5. Huit serre-flancs assurent la tension du tissu (Fig. 5). La rigidité de traction est de $1000 \mathrm{~N} /$ fils et le moment de cisaillement dans le plan est donnée sous forme polynomiale $\mathrm{M}_{\mathrm{s}}(\gamma)=0.37044 \gamma+0.84105 \gamma^{3}+1.03113 \gamma^{5}$. Fig. 5b présente la forme déformée calculée avec la seule raideur en tension. Aucun plissement n'apparaît et les angles de cisaillement sont très importants. Ils atteignent $70^{\circ}$. Figure $5 \mathrm{c}$ et $5 \mathrm{~d}$ montrent la forme déformée calculée lorsque toutes 
les énergies de l'équation 1 sont prises en compte. La raideur en flexion est de 0,15 Nmm dans la figure $5 \mathrm{c}$, et de $15 \mathrm{Nmm}$ dans le cas de la figure $5 \mathrm{~d}$. Il y a de nombreux plissement dans les deux cas et particulièrement entre les serre-flancs. Cependant ces plis sont moins nombreux et leurs tailles sont plus importantes avec l'augmentation de la raideur en flexion (Fig. 5d).

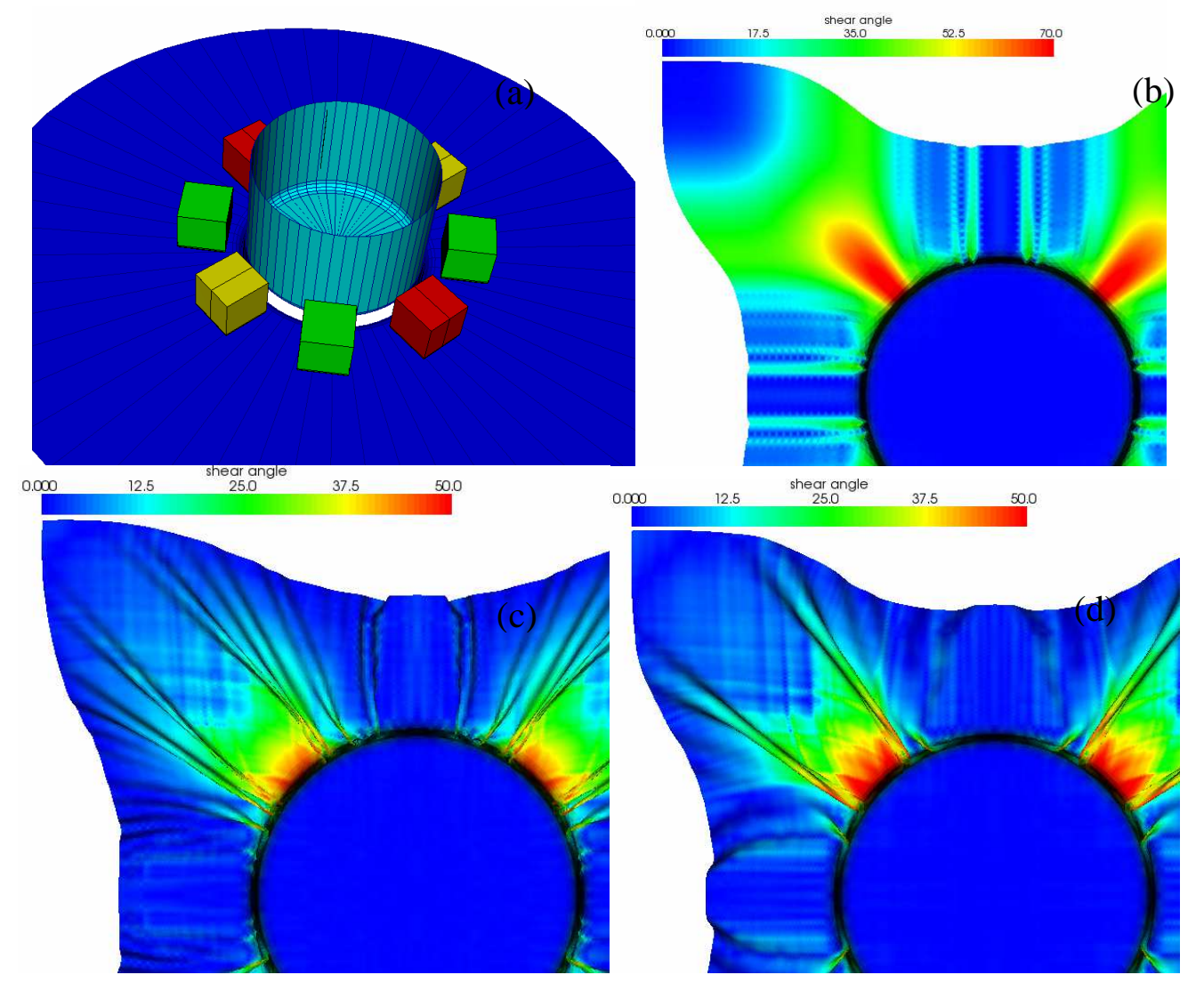

Fig.5. Mise en forme à l'aide d'un poinçon cylindrique, (a)géométrie des outils,(b)raideur en tension seule,(c) faible raideur de flexion, (d)raideur en flexion plus importante.

\subsection{Analyse des plissements lors du formage des multicouches.}

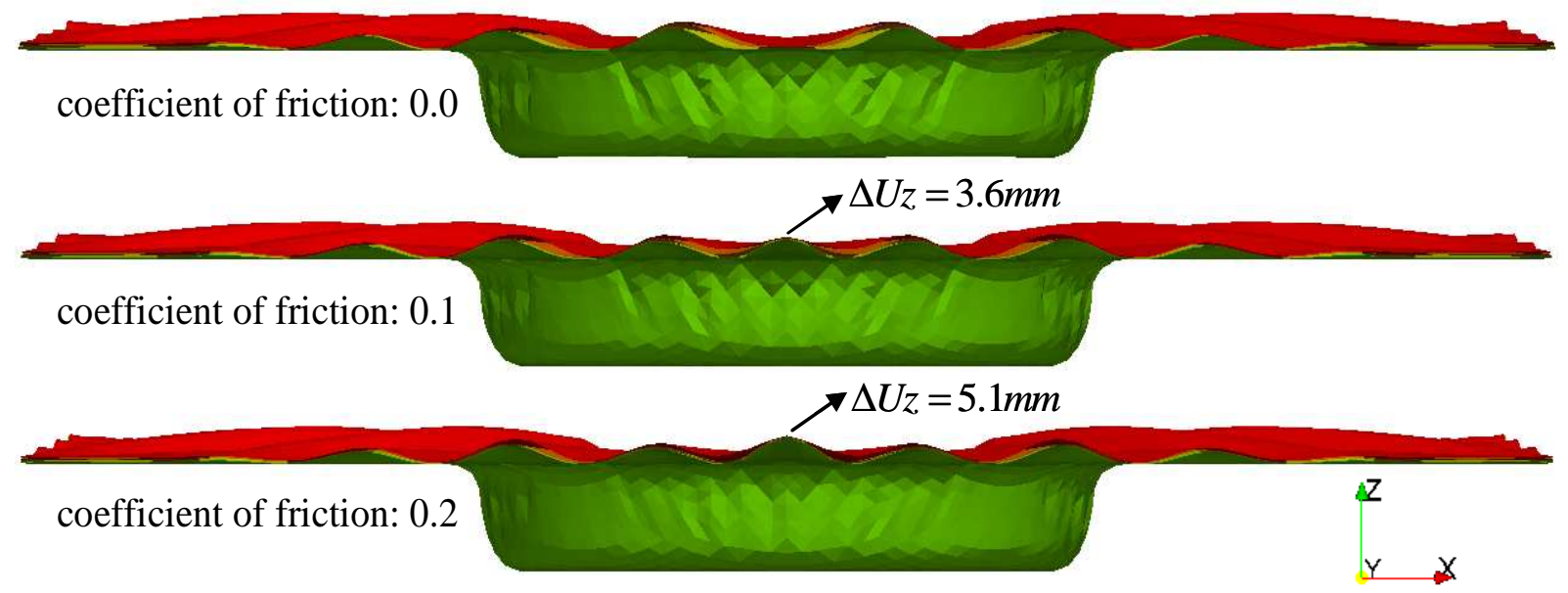

Fig. 6.Evoution de la taille des plis en fonction du coefficient de frottement.

Les simulations proposées reprennent les mêmes géométries et les mêmes caractéristiques mécaniques que dans le précédent cas étudié, à la différence que la course du poinçon est plus courte et que les orientations de mèches changent. Dans le premier cas un empilement de quatre couches orienté à $0^{\circ} / 90^{\circ}$ est embouti en prenant trois coefficient de frottement différents (Fig.6). 
L'exemple fait apparaître que plus le coefficient de frottement est grand plus la taille des plissements est importante. Le second exemple d'empilement (Fig. 7) met en avant l'influence des différentes séquences d'orientations de mèches. Quatre séquences sont proposées : $\left[0^{\circ} / 90^{\circ}, 0^{\circ} / 90^{\circ}\right]_{\mathrm{s}}$ (Fig. 7a), $\left[-30^{\circ} / 60^{\circ},-30^{\circ} / 60^{\circ}\right]_{\mathrm{s}}$ (Fig. 7b), $\left[0^{\circ} / 90^{\circ},-30^{\circ} / 60^{\circ}\right]_{\mathrm{s}}$ (Fig. 13c), et $\left[0^{\circ} / 90^{\circ},-30^{\circ} / 60^{\circ}\right]_{2}$ (Fig. 7d). L'amplitude et la forme des plissements des multicouches changent certes à cause de l'orientation des mèches (Fig. 7a), mais l'ordre de cet empilement joue également un rôle important sur leurs formes.

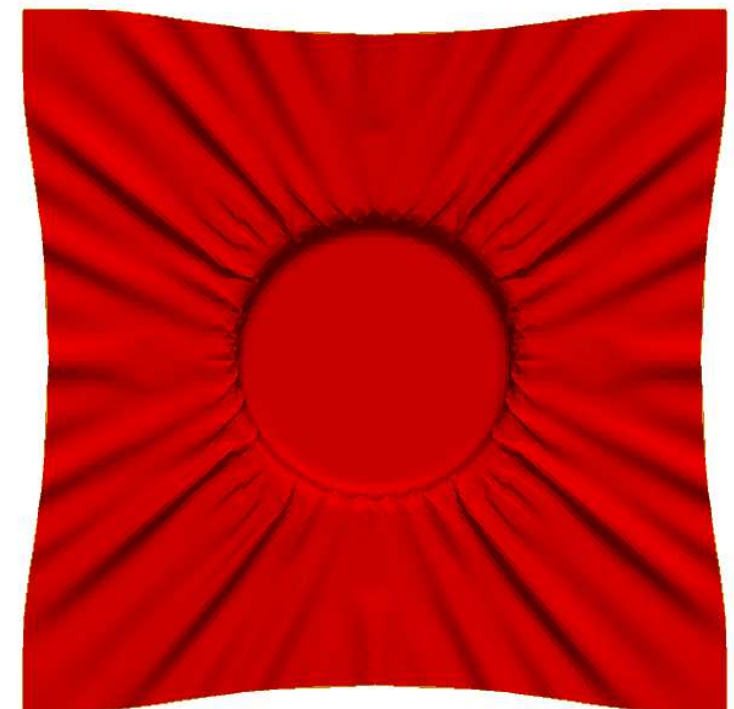

(a) $\left[0 \% 90^{\circ}, 0 \% 909\right.$

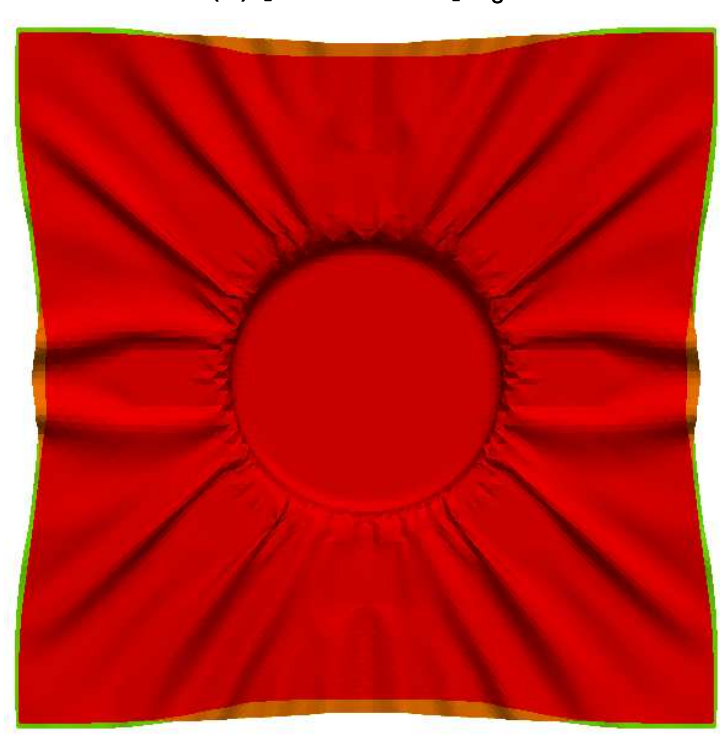

(c) $\left[0 \% 90^{\circ},-30 \% 609 \mathrm{~s}\right.$

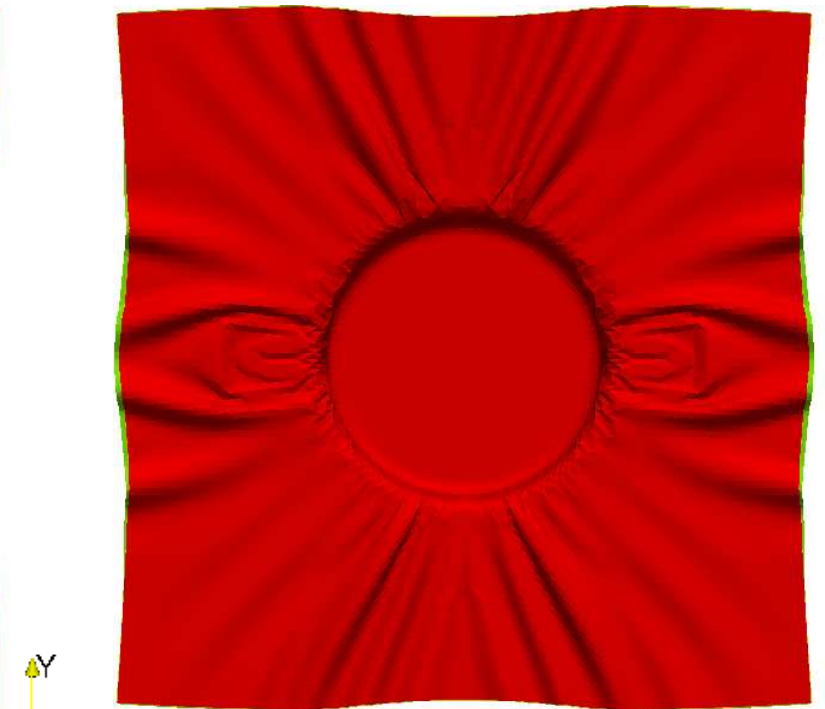

(b) $\left[-30 \% 60^{\circ},-30 \% 609 \mathrm{~s}\right.$

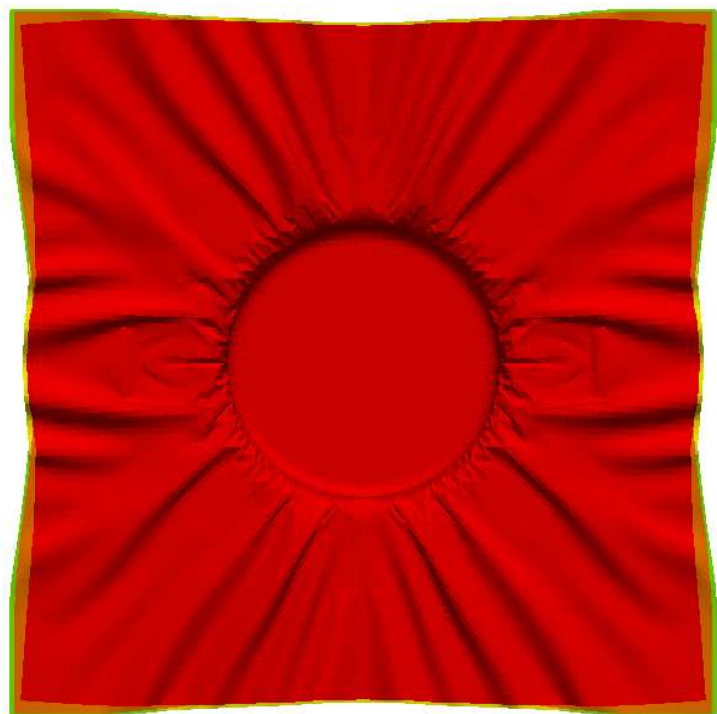

(d) $\left[0 \% 90^{\circ},-30 \% 60\right]_{2}$

Fig. 7. Mise en valeur de la forme des plis en fonction des séquences d'orientation des multicouches. 


\section{Références}

[1] Advani SG. «Flow and rheology in polymeric composites manufacturing ». Elsevier, 1994.

[2] Rudd CD., Long AC. «Liquid Molding Technologies ». Cambridge: Woodhead Pub. Lim., 1997.

[3] De Luca P., Pickett AK. « Numerical and experimental investigation of some press forming parameters of two fibre reinforced thermoplastics: APC2-AS4 and PEI-CETEX ». Composites Part A, Vol. 29, pp. 101-110, 1998.

[4] Hsiao SW., Kikuchi N. « Numerical analysis and optimal design of composite thermoforming process ». Comp. Meth Appl Mech Engrg, Vol.177, pp. 1-34, 1999.

[5] Pickett AK. «Review of finite element methods applied to manufacturing and failure prediction in composite structures ». Applied Composite Material, Vol. 9, pp. 43-58, 2002.

[6] Boisse P. «Finite element analysis of composite forming ». In: Long AC, editor. Composite forming technologies. Woodhead Publishing Limited, 2007.

[7] Hill R., « A general theory of uniqueness and stability in elastic-plastic solids ». J Mech Phys Solids, Vol. 8, pp. 236-249, 1958.

[8] Cao J., Boyce M. «Wrinkle behavior of rectangular plates under lateral constraint ». Int J of Solids Structures, Vol. 34 (2), pp. 153-176, 1997.

[9] Friedl N., Rammerstorfer FG., Fischer FD. «Buckling of stretched strips ». Comp \& Struct, Vol. 78 (1-3), pp. 185-190, 2000

[10] Cerda E., Mahadevan L. « Geometry and Physics of Wrinkling ». Physical review letters, Vol. 90 (7), pp. $074302-$ $1-4,2003$.

[11] Wang CT., Kinzel G., Altan T. «Wrinkling criterion for an anisotropic shell with compound curvatures in sheet forming ». Int J of Mech Sciences, Vol.36, pp. 945-60,1994.

[12] Roddeman DG., Druker J., Oomens CWJ., Janssen JD. « The wrinkling of thin membranes: part 1 - theory, part 2 - numerical analysis ». Transactions of ASME, Vol. 54, pp. 884-892, 1987.

[13] Prodromou AG., Chen J. « On the relationship between shear angle and wrinkling of textile composite preforms ». Composite Part A ,Vol. 28A, pp. 491-503, 1997.

[14] Rozant O., Bourban PE., Manson JAE. « Drapability of dry textile fabrics for stampable thermoplastic preforms ». Composites: Part A, Vol. 31, pp. 1167-1177, 2000.

[15] Potter K. «Bias extension measurements on cross-plied unidirectional prepreg. Composites Part A, Vol. 33, pp. 6373, 2002.

[16] Lebrun G., Bureau MN., Denault J. « Evaluation of bias-extension and picture-frame test methods for the measurement of intraply shear properties of PP/glass commingled fabrics ». Compos Struct, Vol. 61, pp. 341-52, 2003.

[17] Sharma S.B., Sutcliffe M.P.F., Chang S.H. « Characterisation of material properties for draping of dry woven composite material ». Composites Part A, Vol.34, pp. 1167-1175, 2003.

[18] B. Zhu, T.X. Yu, J. Teng, and X.M. Tao, « Theoretical Modeling of Large Shear Deformation and Wrinkling of Plain Woven Composite ». Journal of Composite Materials, Vol. 43,pp. 125 - 138, 2009.

[19] Long AC., Rudd CD. «A simulation of reinforcement deformation during the production of preform for liquid moulding processes ». IMechE J Eng Manuf, Vol. 208, pp. 269-278, 1994.

[20] Wang J., Paton R., Page JR. « The draping of woven fabric preforms and prepregs for production of polymer composite components ». Composites Part A, Vol. 30 (6), pp. 757-765, 1999.

[21] Potluri P., Sharma S., Ramgulam R. « Comprehensive drape modelling for moulding 3D textile preforms ». Composites Part A, Vol. 32 (10), pp. 1415-1424, 2001.

[22] Skordos AA., Monroy Aceves C., Sutcliffe MPF. « A simplified rate dependent model of forming and wrinkling of pre-impregnated woven composites ». Composites: Part A, Vol.38, pp. 1318-1330, 2007.

[23] Cherouat A., Billoet JL. « Mechanical and numerical modelling of composite manufacturing processes deepdrawing and laying-up of thin pre-impregnated woven fabrics ». Journal of Materials Processing Technology, Vol. 118, pp. 460-471, 2001.

[24] H. Lin, J. Wang, A.C. Long, M.J. Clifford, P. Harrison, « Predictive modelling for optimization of textile composite forming ». Composites Science and Technology, Vol. 67, pp. 3242-3252, 2007.

[25] J. Lee, S. Hong, W. Yu, T. Kang, « The effect of blank holder force on the stamp forming behaviour of non-crimp fabric with a chain stitch ». Compos. Sci Tech, Vol. 67(3-4), pp. 357-366, 2007.

[26] Kawabata S., Niwa M., Kawai H. « The Finite Deformation Theory of Plain Weave Fabrics Part I: The Biaxial Deformation Theory ». Journal of the Textile Institute, Vol. 64(1), pp. 21-46, 1973.

[27] Buet-Gautier K., Boisse P. « Experimental analysis and modeling of biaxial mechanical behavior of woven composite reinforcements ». Experimental Mechanics, Vol. 41 (3), pp. 260-269, 2001.

[28] Carvelli V., Corazza C., Poggi C. « Mechanical modelling of monofilament technical textiles ». Computational Materials Science, Vol. 42, pp. 679-691, 2008. 
[29] Willems A., Lomov SV., Verpoest I., Vandepitte D. «Optical strain fields in shear and tensile testing of textile reinforcements ». Composites Science and Technology, Vol. 68, pp. 807-819, 2008.

[30] Peng XQ., Cao J., Chen J., Xue P., Lussier DS., Liu L. « Experimental and numerical analysis on normalization of picture frame tests for composite materials ». Compos Sci Tech, Vol. 64, pp. 11-21, 2004.

[31] Harrison P., Clifford MJ., Long AC. «Shear characterisation of viscous woven textile composites: a comparison between picture frame and bias extension experiments ». Compos Sci Tech, Vol. 64, pp.1453-1465, 2004.

[32] Potluri P., Perez Ciurezu DA., Ramgulam RB. « Measurement of meso-scale shear deformations for modelling textile composites ». Composites Part A, Vol. 37, pp. 303-314, 2006.

[33] Lomov SV., Willems A., Verpoest I., Zhu Y., Barburski M., Stoilova Tz. « Picture frame test of woven composite reinforcements with a full-field strain registration ». Textile Research Journal, Vol. 76 (3), pp. 243-252, 2006.

[34] Launay J., Hivet G., Duong AV., Boisse P. « Experimental analysis of the influence of tensions on in plane shear behaviour of woven composite reinforcements ». Compos Sci Tech, Vol. 68, pp. 506-515, 2008.

[35] Lomov S., Boisse P., Deluycker E., Morestin F., Vanclooster K., Vandepitte D., Verpoest I., Willems A. « Full field strain measurements in textile deformability studies ». Composites: Part A, Vol. 39, pp. 1232-1244, 2008.

[36] Cao J., Akkerman R., Boisse P., Chen J. et al. « Characterization of Mechanical Behavior of Woven Fabrics: Experimental Methods and Benchmark Results ». Composites Part A, Vol. 39, pp.1037-1053, 2008.

[37] Kawabata S. «The Standardization and Analysis of Hand Evaluation ». Osaka: The Textile Machinery Society of Japan, 1986.

[38] Lahey TJ., Heppler GR. « Mechanical Modeling of Fabrics in Bending ». ASME Journal of Applied Mechanics, Vol. 71, pp. 32-40, 2004.

[39] de Bilbao E, Soulat D, Hivet G, Gasser A., « Experimental Study of Bending Behaviour of Reinforcements ». Experimental Mechanics, Vol. 50, pp. 333-351, 2010.

[40] Hamila N. «Simulation de la mise en forme des renforts composites mono et multiplis ». Thèse de doctorat, Institut National des Sciences Appliquées, 2007.

[41] Hamila N., Boisse P., Sabourin F., Brunet M. « A semi-discrete shell finite element for textile composite reinforcement forming simulation ». Int J Numerical Methods in Engineering, Vol. 79, pp. 1443-1466, 2009.

[42] Onate E., Zarate F. « Rotation-free triangular plate and shell elements ». Int J for Num Meth in Eng, Vol. 47, pp. 557-603, 2000.

[43] Sabourin F., Brunet M. «Detailed formulation of the rotation-free triangular element "S3" for general purpose shell analysis ». Engineering computations, Vol. 23 (5), pp. 469-502, 2006.

[44] Daniel JL., Soulat D., Dumont F., Zouari B., Boisse P., Long AC. « Forming simulation of very unbalanced woven composite reinforcements ». Int J of Forming Processes, Vol. 6 (3-4), pp. 465-480, 2003. 